\title{
On the binary expansions of algebraic numbers
}

\author{
par D. H. BAILEY, J. M. BORWEIN, R. E. CRANDALL \\ et C. POMERANCE
}

RÉSUMÉ. En combinant des concepts de thorie additive des nombres avec des rsultats sur les dveloppements binaires et les sries partielles, nous tablissons de nouvelles bornes pour la densit de 1 dans les dveloppements binaires de nombres algbriques rels. Un rsultat clef est que si un nombre rel $y$ est algbrique de $\operatorname{degr} D>1$, alors le nombre $\#(|y|, N)$ de 1 dans le dveloppement de $|y|$ parmi les $N$ premiers chiffres satisfait

$$
\#(|y|, N)>C N^{1 / D}
$$

avec un nombre positif $C$ (qui dpend de $y$ ), la minoration tant vraie pour tout $N$ suffisamment grand. On en dduit la transcendance d'une classe de nombres rels $\sum_{n \geq 0} 1 / 2^{f(n)}$ quand la fonction $f$, valeurs entires, crot suffisamment vite, disons plus vite que toute puissance de $n$. Grce ces mthodes on redmontre la transcendance du nombre de Kempner-Mahler $\sum_{n \geq 0} 1 / 2^{2^{n}}$; nous considrons galement des nombres ayant une densit sensiblement plus grande de 1 . Bien que le nombre $z=\sum_{n \geq 0} 1 / 2^{n^{2}}$ ait une densit de 1 trop grande pour que nous puissions lui appliquer notre rsultat central, nous parvenons dvelopper une analyse fine de thorie des nombres avec des calculs tendus pour rvler des proprits de la structure binaire du nombre $z^{2}$.

Abstract. Employing concepts from additive number theory, together with results on binary evaluations and partial series, we establish bounds on the density of 1's in the binary expansions of real algebraic numbers. A central result is that if a real $y$ has algebraic degree $D>1$, then the number \# $(|y|, N)$ of 1-bits in the expansion of $|y|$ through bit position $N$ satisfies

$$
\#(|y|, N)>C N^{1 / D}
$$

Bailey's work is supported by the Director, Office of Computational and Technology Research, Division of Mathematical, Information, and Computational Sciences of the U.S. Department of Energy, under contract number DE-AC03-76SF00098.

Borwein's work is funded by NSERC and the Canada Research Chair Program. 
for a positive number $C$ (depending on $y$ ) and sufficiently large $N$. This in itself establishes the transcendency of a class of reals $\sum_{n>0} 1 / 2^{f(n)}$ where the integer-valued function $f$ grows sufficiently fast; say, faster than any fixed power of $n$. By these methods we re-establish the transcendency of the Kempner-Mahler number $\sum_{n \geq 0} 1 / 2^{2^{n}}$, yet we can also handle numbers with a substantially denser occurrence of 1 's. Though the number $z=$ $\sum_{n \geq 0} 1 / 2^{n^{2}}$ has too high a 1 's density for application of our central result, we are able to invoke some rather intricate numbertheoretical analysis and extended computations to reveal aspects of the binary structure of $z^{2}$.

\section{Introduction}

Research into the statistical character of digit expansions is often focused on the concept of normality. We call a real number $b$-normal if its base- $b$ digits are random in a certain technical sense (see [31], [21], [3], and references therein). Qualitatively speaking, $b$-normality requires every string of $k$ consecutive base- $b$ digits to occur, in the limit, $1 / b^{k}$ of the time, as if the digits are generated by tossing a "fair" $b$-sided die. In spite of the known fact that almost all numbers are $b$-normal (in fact almost all are absolutely normal, meaning $b$-normal for every base $b=2,3, \ldots$ ) not a single, shall we say "genuine" fundamental constant such as $\pi, e, \log 2$ is known to be $b$-normal for any $b$. Artificially constructed normals are known, such as the 2-normal binary Champernowne number [9]

$$
C_{2}=(0.11011100101110 \ldots)_{2},
$$

obtained by sheer concatenation of the binary of positive integers. Previous research that motivates the present work includes [3], where a certain "Hypothesis A" relevant to chaotic maps is shown to imply 2-normality of $\pi, \log 2, \zeta(3)$; and [4], where the historical work of Korobov, Stoneham and others is augmented to establish $b$-normality of, shall we say, "less artificial" constants such as the numbers $\sum_{n \geq 0} 1 /\left(c^{n} b^{c^{n}}\right)$ where $b, c>1$ are coprime. Intriguing connections with yet other fields - such as ergodic theory - are presented in [22].

Of interest for the present work is that all real algebraic irrationals are widely believed - shall we say suspected - to be absolutely normal (and this belief is at least a half-century old; see for example $[6,7]$ ). This suspicion is based on numerical and visual evidence that the digit expansions of algebraics do appear empirically "random." Yet again, the mathematical situation is as bleak as can be: Not a single algebraic real is known to be $b$-normal, nor has a single algebraic real irrational been shown not to be $b$-normal; all of this regardless of the base $b$. Though we expect every irrational algebraic real is absolutely normal, for all we know it could even 
be that some algebraics are absolutely abnormal, i.e. not $b$-normal for any $b$ whatsoever (absolutely abnormal numbers do exist; see [28]).

Herein we focus on the binary scenario $b=2$, and though we do not achieve normality results per se, we establish useful lower bounds on the occurrence of 1-bits in positive algebraics. Our central result is that if $y$ is a real algebraic of degree $D>1$, then there exists a positive number $C$ (depending only on $y$ ) such that for sufficiently large $N$ the number $\#(|y|, N)$ of 1's in the binary expansion of $|y|$ through the $N$-th bit position satisfies

$$
\#(|y|, N)>C N^{1 / D} .
$$

To achieve this bound we borrow ideas from additive number theory; in particular we employ the notion of additive representations. This notion is combined with our own bounds on the count of 1-bits resulting from binary operations, and also with previous observations on "BBP tails" that arise from arbitrary left-shifts of infinite series. In Section 6 we define and elaborate on BBP tails.

Irrational numbers $y$ for which \# $(|y|, N)$ cannot achieve the above bound for any degree $D$ are necessarily transcendental. In this way we easily reestablish the transcendency of the Kempner-Mahler number

$$
M=\sum_{n \geq 0} \frac{1}{2^{2^{n}}},
$$

first shown to be transcendental by Kempner [19], but the transcendency cannot be established directly from the celebrated Thue-Siegel-Roth theorem on rational approximations to algebraics (there are interesting anecdotes concerning Mahler's approach to such an impasse, including his results on $p$-adic Thue-Siegel theory and his functional methods; see [26, 27, 29]). Incidentally, the number $M$ above is sometimes called the Fredholm number, but this attribution may be historically erroneous [35]. (See also [1] for more on the number $M$.)

We can also handle numbers having a higher density of 1's than does $M$. For example, by our methods the Fibonacci binary

$$
X=\sum_{n \geq 0} \frac{1}{2^{F_{n}}}
$$

having 1's at Fibonacci-number positions $0,1,1,2,3,5 \ldots$ is transcendental. Now $X$ was proved transcendental some decades ago [27] and explicit irrationality measures and certain continued-fraction properties are known for $X$ [36]. In the present treatment, we can handle numbers like $X$ but where the growth of the exponents is more general than the classic growth of the $F_{n}$. 
With our central result we establish the transcendency of numbers whose 1-bits are substantially more dense than in the above examples, an example of such a "denser" number being

$$
\sum_{n \geq 3} \frac{1}{2^{\left\lfloor n^{\log \log n\rfloor}\right.}}
$$

Incidentally, in the late stages of the present research project we found that this notion of "digital thinking" to establish results in analysis had been foreshadowed by a specific, pedagogical proof by M. Knight [20] that for any base $b>1$

$$
\sum_{n \geq 0} \frac{1}{b^{2^{n}}}
$$

is transcendental (note that $b=2$ gives the number $M$ above). The author used terms such as "islands" for flocks of digits guarded on both sides by enough zeros to avoid carry problems when integral powers of a real number are taken. As will be seen, such notions pervade also our own treatment; however our results pertain to general 1-bit densities and not to specific real numbers. Other historical foreshadowings of our approach exist [34] [25]. (See also our Section 11 on open problems.)

Aside from transcendency results, we can employ the central theorem to establish bounds on the algebraic degree. For example, we shall see that

$$
\sum_{n \geq 0} \frac{1}{2^{n^{k}}}, \quad \sum_{p \text { prime }} \frac{1}{2^{p^{k}}}
$$

must have algebraic degrees at least $k, k+1$ respectively. (In this context we think of a transcendental number as having infinite degree.) Thus for example, $\sum 1 / 2^{p^{2}}$ must be an at-least-cubic irrational.

There are interesting numbers that do not fall under the rubric of our central theorem, such as the "borderline" case:

$$
z=\sum_{n \geq 0} \frac{1}{2^{n^{2}}}=\frac{1}{2}\left(1+\theta_{3}\left(\frac{1}{2}\right)\right),
$$

where $\theta_{3}$ is the standard Jacobi theta function. The problem is that $\#(z, N) \sim \sqrt{N}$, so our central theorem does not give any information on the algebraic degree of $z$. Yet we are able to use further number-theoretical analysis - notably the theory of representations of integers as sums of two squares - to establish quadratic irrationality for $z$. We further argue, on the basis of such analysis, that $z^{2}$ has almost all 0's, and more precisely that the 1's count through the $N$-th bit position has a certain asymptotic behavior. Incidentally the number $z$, being essentially the evaluation of a theta function at an algebraic argument, is known to be transcendental 
by other methods $[30,5,12]$. We stress that our binary approach is an apparently new way to look at such issues.

\section{Additive representations}

For any real nonnegative number $x$ we consider the binary expansion

$$
x=\left(\ldots x_{-3} x_{-2} x_{-1} x_{0} \cdot x_{1} x_{2} x_{3} \ldots\right)_{2} .
$$

The assignment of (finitely many) nonpositive indices for bits $x_{i}$ to the left of the decimal (or if you will, binary) point is a convenience, for we shall, of course, be concentrating a great deal on the bits to the right. We adopt the convention that no $x$ can end with infinitely many successive 1's, and this forces uniqueness of the binary expansion. Next we denote the 1's-position set of $x$ by

$$
\mathcal{P}(x)=\left\{p: x_{p}=1\right\},
$$

and further define $r_{1}(x, p)=1$ if $x_{p}=1$, else 0 . (The rationale for the notation " $r_{1}$ " will be momentarily evident.) Now the number of 1-bits through bit position $N$ inclusive is

$$
\#(x, N)=\sum_{m \leq N} r_{1}(x, m)=\sum_{p \in \mathcal{P}(x), p \leq N} 1 .
$$

Note that when $x$ is a nonnegative integer, $\#(x, 0)$ is the number of 1 's in $x$. So for example $\#(7,0)=3$. On the other hand, for $x=\sqrt{2}=$ $(1.011010100 \ldots)_{2}$, say, we have $\#(x, 0)=1, \#(x, 5)=4$, and so on.

We next introduce the representation count

$$
r_{d}(x, n)=\#\left\{\left(p_{1}, \ldots, p_{d}\right) \in \mathcal{P}^{d}: p_{1}+\cdots+p_{d}=n\right\},
$$

just as in additive number theory where one studies representations of integers $n$ as sums of primes, or squares, and so on. It is evident that $r_{d}$ can be expressed as an acyclic convolution:

$$
r_{d}(x, n)=\sum_{i+j=n} r_{d-1}(x, i) r_{1}(x, j) .
$$

We shall also employ a step-function on integers $r$, namely $H(r)=1$ if $r>$ 0 , else 0 . Thus $H\left(r_{d}(n)\right)=1$ signifies that $n$ has at least one representation $p_{1}+\cdots+p_{d}$. For our analysis it is a simple but useful combinatorial observation that the count of representables, call it

$$
\rho_{d}(N)=\sum_{n \leq N} H\left(r_{d}(x, n)\right),
$$

satisfies

$$
\rho_{d}(N) \leq \sum_{n \leq N} r_{d}(x, n) \leq \#(x, N)^{d} .
$$


Also of use will be an attractive relation for positive integral powers of $x$ :

$$
x^{d}=\sum_{n} \frac{r_{d}(x, n)}{2^{n}} .
$$

Unfortunately it is in general extremely difficult to convert partial knowledge of the representation sequence $\left(r_{d}(x, n)\right)$ into precise results on the binary expansion of $x^{d}$. The problem is that of carry: A summand $r_{d}(x, n) / 2^{n}$ possibly causes carry, about $\lg r_{d}$ positions to the left, and thus the summands interfere (herein $\lg x$ means the base- 2 logarithm of $x$ ). It can be said that the goal of the present treatment is the circumvention of this carry problem.

An instructive digression is appropriate here. With a view to additive number theory, let us define the number

$$
G=\sum_{p \text { prime, odd }} \frac{1}{2^{(p-1) / 2}}=(0.11101101 \ldots)_{2} .
$$

Note that $\#(G, N)=\pi(2 N+2)-1$, where $\pi$ is the standard prime-counting function. Then $r_{2}(G, N)$ is precisely the number of representations of $2 N+2$ as a sum of two odd primes. Even if we knew the truth of the Goldbach conjecture - in this scenario, that every $N>2$ has $H\left(r_{2}(x, N)\right)=1$-we would still not immediately know the binary expansion of $G^{2}$, because of the carry problem. For all we know, it could be that the question of irrationality for $G^{2}$ is more difficult than the Goldbach conjecture itself. Conversely, it is unclear whether complete knowledge of the binary expansion of $G^{2}$ would yield results on the celebrated conjecture. In fact, it is easy to see that $r_{2}(G, N)$ is unbounded, so arbitrarily long carries (deposition of bits arbitrarily far to the left of a given position) can be expected.

Similarly, for the number $z=\sum_{n \geq 0} 2^{-n^{2}}$ introduced earlier we know that $z^{4}$ has a representation sequence $\left(r_{4}\left(z^{4}, 0\right), r_{4}\left(z^{4}, 1\right), \ldots\right)$ of all positive entries, on the basis of the Lagrange theorem that every nonnegative integer is a sum of four squares. Here again, little can be gleaned about the binary expansion of $z^{4}$ from this perspective, again because of carry. We study the number $z$ further in Section 9 .

Now back to positive powers of $x$ and representation lists. A sum we later call a "tail component" defined

$$
T_{d}(x, R)=\sum_{m \geq 1} \frac{r_{d}(x, R+m)}{2^{m}},
$$

which we note is $2^{R}$ times a partial series for the power $x^{d}$, can be bounded via combinatorial observations, as in 
Theorem 2.1. For $x \in(1,2)$ (whether algebraic or not) and $d \geq 1$ we have

$$
r_{d}(x, n) \leq\left(\begin{array}{c}
n+d-1 \\
d-1
\end{array}\right) .
$$

Moreover, for the sum $T_{d}$ defined above, we have for $0 \leq R \leq N$ the upper bound

$$
T_{d}(x, R)<\frac{(R+d)^{d}}{(d-1) !(R+1)} \leq \frac{(N+d)^{d}}{(d-1) !(N+1)}
$$

and the average bound

$$
\sum_{0 \leq R \leq N} T_{d}(x, R)<\frac{(N+d)^{d}}{(d-1) !} .
$$

Proof. From the convolution

$$
r_{d}(x, n)=\sum_{i+j=n} r_{d-1}(x, i) r_{1}(x, j)
$$

we have

$$
r_{d}(x, n) \leq \sum_{i_{1}, \ldots, i_{d} \in[0, n], \sum i_{j}=n} 1=\left(\begin{array}{c}
n+d-1 \\
d-1
\end{array}\right) .
$$

Thus $T_{d}(x, R) \leq U_{d}(R)$ where

$$
U_{d}(R)=\sum_{m \geq 1} \frac{1}{2^{m}}\left(\begin{array}{c}
R+m+d-1 \\
d-1
\end{array}\right) .
$$

This expression is seen to satisfy the recurrence relation

$$
U_{d}(R)=2 U_{d-1}(R)+\left(\begin{array}{c}
R+d-1 \\
d-1
\end{array}\right),
$$

which can be used to establish a finite form for $U_{d}$ :

$$
U_{d}(R)=\sum_{j=0}^{d-1}\left(\begin{array}{c}
R+d \\
j
\end{array}\right) \text {. }
$$

So we have

$$
U_{d}(R)<\frac{(R+d)^{d-1}}{(d-1) !} \sum_{n \geq 0}\left(\frac{d-1}{R+d}\right)^{n}=\frac{(R+d)^{d}}{(d-1) !(R+1)} .
$$

Thus, the first bound follows. The bound on the sum $\sum T_{d}$ is simply obtained by summing the first bound over the stated range of $R$.

Remark. The finite form for $U_{d}(R)$ noted above is a polynomial in $R$ with nonnegative coefficients and with main term $R^{d-1} /(d-1)$ !, so that this expression is not only a lower bound for $U_{d}(R)$, but is also equal to 
it asymptotically. Moreover, it is possible to express $U_{d}(R)$ as a hypergeometric integral:

$$
U_{d}(R)=\frac{(R+d) !}{R !(d-1) !} \int_{0}^{1}(2-x)^{d-1} x^{R} d x .
$$

We admit that the bounds of Theorem 2.1 and the present remark are actually stronger than what we need here; however, such stronger bounds could be useful in future research.

\section{Preliminary bound on 1's density}

Let $x$ be a real algebraic irrational. The Thue-Siegel-Roth theorem [33] says that for any $\epsilon>0$ the inequality

$$
\left|x-\frac{a}{b}\right|<\frac{1}{b^{2+\epsilon}}
$$

has at most finitely many integer-pair solutions $a, b$. This means that the 1-bits of such an $x$ cannot be too far apart, in the sense of

Theorem 3.1. For a real positive algebraic irrational number $x$, and any $\delta>0$, the 1's positions $p_{i} \in \mathcal{P}(x)$ satisfy, for sufficiently large $m$,

$$
p_{m}<(2+\delta) p_{m-1} \text {. }
$$

Furthermore, for sufficiently large $k$, the interval

$$
\left(\left\lfloor\frac{k}{2+\delta}\right\rfloor, k\right)
$$

always contains a 1's position. Finally, the 1's count through sufficiently large position $n$ satisfies

$$
\#(x, n)>(1-\delta) \lg n .
$$

Proof. When $x$ is irrational, $\mathcal{P}(x)$ is an infinite set, so arbitrarily large $p_{i}$ can be chosen, with

$$
x-\sum_{p \in \mathcal{P}(x), p \leq p_{i}} \frac{1}{2^{p}}<\frac{2}{2^{p_{i+1}}} .
$$

Now the sum is a rational $a / b$ with $b=2^{p_{i}}$, and so the first inequality of the theorem is clearly satisfied if $p_{i}$ is large enough. The rest of the conclusions are immediate from said inequality.

The bound $\#(x, n)>(1-\delta) \lg n$ is admittedly weak, relative to what we aim to prove later. It does, however, establish the transcendency of any number

$$
m_{\alpha}=\sum_{n \geq 0} \frac{1}{2^{\left\lfloor\alpha^{n}\right\rfloor}}
$$


for any real $\alpha>2$. Note that the Kempner-Mahler number $M=m_{2}=$ $\sum_{n>0} 1 / 2^{2^{n}}$ lies just out of reach of the Thue-Siegel-Roth implications. We shall be able to use our binary approach to establish, in fact, the transcendency of $m_{\alpha}$ for any real $\alpha>1$.

There is a curious aspect to Theorem 3.1, namely, however weak the bounds on 1's counts may be, there is a crucial juncture in what follows (the central Theorem 7.1) where we need Theorem 3.1 to assail the ubiquitous problem of carry propagation.

\section{Bounds for binary evaluations}

For nonnegative integers $n$ we have defined $\#(n, 0)$ as the number of 1 's in the binary expansion of $n$. We proceed to give convexity relations on binary evaluations, i.e. on sums and products of integers, starting with some simple observations:

Lemma 4.1. For integers $n>0, j \geq 0$, we have

$$
\begin{gathered}
\#(n, 0) \leq 1+\lg n \leq n, \\
\#\left(2^{j} n, 0\right)=\#(n, 0), \\
\#\left(n+2^{j}, 0\right)=\#(n, 0)+1-k_{j},
\end{gathered}
$$

where in the last relation $k_{j}$ is the number of consecutive 1's in $n$ counting from the $(-j)$-th position inclusive, to the left.

Proof. The first inequality follows from the observation that the total number $B(n)$ of bits in $n$ (counting 0 's and 1's) satisfies $2^{B(n)-1} \leq n$, and $\#(n, 0) \leq B(n)$. The second statement is obvious (left-shifting by $j$ bits introduces no new 1's). The third statement follows by the simple rule of add-with-carry.

This lemma leads to

Theorem 4.2 (Convexity relations). For nonnegative integers $m, n$ we have upper bounds on the 1's counts of evaluations, as

$$
\begin{gathered}
\#(m+n, 0) \leq \#(m, 0)+\#(n, 0), \\
\#(m n, 0) \leq \#(m, 0) \#(n, 0) .
\end{gathered}
$$

Proof. The first, additive relation follows by repeated application of the last equality of Lemma 4.1, one application for each 1-bit of $m$, say. The second, multiplicative relation follows in similar fashion, by writing $m n=$ $\left(\sum 2^{-p}\right) n$, where $p$ runs through the 1's positions of $m$, and using the second (shift) relation of Lemma 4.1. 
It would greatly enhance the present study if we could obtain lower bounds on the 1's counts of binary evaluations. The extreme difficulty of such a program can be exemplified in several ways. Consider the famous factorization of the 7 -th Fermat number, namely $2^{128}+1$, but expressed in binary:

100000000000000000000000000000000000000000000000000000000000 $000000000000000000000000000000000000000000000000000000000000000000001=$

$11010011111010101111110000111010111100010100011000000001 \times$ 1001101010100000001110111010110110100100011001100001100101011101000000001

which display dashes any hope of a useful, unconditional lower bound on $\#(m n, 0)$. Also interesting is this: If a Mersenne number $p=2^{q}-1$, with $q$ prime, say, is a product of two prime factors, say $p=f g$, then the convexity Theorem 4.2 implies \# $(f, 0) \#(g, 0)>\#(p, 0)=q$ (recall $q$ is prime so this \# product cannot be $q$ ), which means that the factors $f, g$ cannot both be too sparse with 1's. For example, $2^{11}-1=23 \cdot 89$ and each factor has four 1's; sure enough $4 \cdot 4>11$.

But for the present study on real numbers, there is a more telling disappointment in regard to lower bounds on 1's densities of products. Consider two sets of integers:

$$
\begin{gathered}
S=\left\{n \geq 0: n_{-2 j}=0\right\}=\{0,2,8,10,32,34,40,42, \ldots\}, \\
T=\left\{n \geq 0: n_{-2 j+1}=0\right\}=\{0,1,4,5,16,17,20,21, \ldots\}=S / 2,
\end{gathered}
$$

so that elements of $S, T$ have 0 -bits in all even, odd positions respectively. Now define associated real numbers:

$$
x_{S}=\sum_{s \in S} \frac{1}{2^{s}}, \quad x_{T}=\sum_{t \in T} \frac{1}{2^{t}} .
$$

It is not hard to show that both of these real-numbers have "square-root" 1 's densities, that is both \# $\left(x_{S}, N\right), \#\left(x_{T}, N\right)$ are roughly of order $\sqrt{N}$, so that both $x_{S}, x_{T}$ are irrational; in fact the sum $x_{S}+x_{T}$ is irrational, since the intersection of the $S, T$ sets is just $\{0\}$ and so there is only one trivial carry for the sum. However, all of this having been said, it turns out amazingly enough that one has rational product

$$
x_{S} x_{T}=2=(10.0000 \ldots)_{2},
$$

which reveals that two numbers each with square-root 1's density can have an extremal 1's density, in this case a zero density because of carry. By looking at the representation counts for integers in $S+S$ one may show $x_{S}^{2}, x_{T}^{2}$, hence (since $2 x_{S} x_{T}=4$ ) also $\left(x_{S}+x_{T}\right)^{2}$ are irrational. In any case, we shall be able to handle certain real numbers whose 1's count is genuinely less than $\sqrt{N}$. 
Back to the manageable case of upper bounds for binary evaluations, consider the polynomial

$$
f(x)=A_{D} x^{D}+A_{D-1} x^{D-1}+\ldots A_{1} x+A_{0},
$$

for integers $A_{i}$ all nonnegative. Then from Lemma 4.1 and Theorem 4.2 we easily have, for nonnegative integers $n$, the following convexity relation for polynomial evaluations:

$$
\#(f(n), 0) \leq \sum_{d=0}^{D} \max \left(0,1+\lg A_{d}\right) \#(n, 0)^{d} \leq f(\#(n, 0)) .
$$

This relation will next be applied to algebraic numbers whose minimum integer polynomial has all coefficients (except $A_{0}$ ) nonnegative.

\section{Application of binary-evaluation bounds}

Our strongest bounds on 1's density will be obtained for the class of real algebraic irrationals for which the coefficients of the minimum integer polynomial are nonnegative, except for the constant term. We begin with

Lemma 5.1. For irrational $x \in(1,2)$ and a given integral power $d$, the inequality

$$
0<x^{d}-\frac{\left\lfloor 2^{N} x\right\rfloor^{d}}{2^{N d}}<\frac{d 2^{d}}{2^{N}}
$$

holds for all sufficiently large $N$.

Proof. Setting $i=\left\lfloor 2^{N} x\right\rfloor$, we have $2^{N} \leq i<2^{N+1}$, and $x=i / 2^{N}+z$, where $z \in\left(0,1 / 2^{N}\right)$. Now

$$
x^{d}=\frac{i^{d}}{2^{N d}}\left(1+2^{N} z / i\right)^{d},
$$

so that

$$
0<x^{d}-\frac{i^{d}}{2^{N d}}<\frac{i^{d}}{2^{N d}}\left((1+1 / i)^{d}-1\right) .
$$

Choose $M$ such that $d<i$ for $N>M$, whence

$$
x^{d}-\frac{i^{d}}{2^{N d}}<\frac{i^{d}}{2^{N d}} \frac{2 d}{i}<(e-1) d \frac{2^{(N+1)(d-1)}}{2^{N d}}<\frac{d 2^{d}}{2^{N}} .
$$

We are now in a position to state

Theorem 5.2. Let $y$ be a real algebraic of degree $D>1$ and assume for $x=|y| / 2^{\lfloor\lg |y|\rfloor}$ a minimum integer polynomial equation

$$
A_{D} x^{D}+A_{D-1} x^{D-1}+\ldots A_{1} x+A_{0}=0,
$$


where $A_{D}>0$ and $A_{D-1}, \ldots, A_{1}$ are nonnegative integers. Then for any $\epsilon>0$ we have

$$
\#(|y|, N)>(1-\epsilon)\left(1+\lg A_{D}\right)^{-1 / D} N^{1 / D}
$$

for sufficiently large $N$ (with threshold depending on $y, \epsilon$ ).

Proof. Note that $x \in(1,2)$ and because $x$ is a shift of $y$, the counts $\#(x, N), \#(y, N)$ differ only by an integer constant, so we may concentrate on $x$. Observe that $A_{0}$ is a negative integer. From Lemma 5.1 we can select $N$ and assign $i=\left\lfloor 2^{N} x\right\rfloor$ so that

$$
x^{d}=\frac{i^{d}}{2^{N d}}+z_{d},
$$

with $z_{d} \in\left(0, d 2^{d} / 2^{N}\right)$, for $1 \leq d \leq D$. Now define the integer

$$
Y_{N}=2^{N D} \sum_{d=1}^{D} A_{d} i^{d} 2^{-N d}=2^{N D} \sum_{d=1}^{D} A_{d}\left(x^{d}-z_{d}\right),
$$

so that

$$
-A_{0}=\frac{Y_{N}}{2^{N D}}+z_{N}
$$

where

$$
0<z_{N}=\sum_{d=1}^{D} A_{d} z_{d}<\frac{1}{2^{N}} \sum_{d=1}^{D} d 2^{d} A_{d} .
$$

(It is this last inequality where the signs of the $A_{i}, i>0$ are essential.) Thus for sufficiently large $N$ we have a fractional part

$$
\left\{\frac{Y_{N}}{2^{N D}}\right\}=\left\{-A_{0}-z_{N}\right\}=\left\{1-z_{N}\right\} \geq 1-\frac{C}{2^{N}}>0
$$

for a strictly positive constant $C=\sum_{d=1}^{D} d 2^{d} A_{d}$ independent of $N$. But this means for some constant $C^{\prime}$ (also independent of $N$ ) that the integer $Y_{N}$ has more than $N-C^{\prime} 1$-bits. Since $\#(i, 0)=\#(x, N)$, on using Theorem 4.2 , we have (again using in an essential way that $A_{i} \geq 0$ for $i>0$ )

$$
\begin{aligned}
N-C^{\prime} & <\#\left(Y_{N}, 0\right) \leq \sum_{d=1}^{D} \max \left(0,1+\lg A_{d}\right) \#(x, N)^{d} \\
& \leq \#(x, N)^{D}\left(\left(1+\lg A_{D}\right)+\frac{A_{D-1}}{\#(x, N)}+\ldots\right),
\end{aligned}
$$

and since $\#(x, N)$ is unbounded ( $x$ is irrational) the result follows.

A side result is 
Corollary 5.3. If $y>0$ is irrational, and there exists an integer $d>1$ such that for every $\eta>0$ we have

$$
\#(y, N)<\eta N^{1 / d}
$$

for infinitely many $N$, then $y^{d}$ is also irrational.

Proof. Assuming $y^{d}$ is rational then for $x=y / 2^{\lfloor\lg y\rfloor}$ there is a polynomial $A x^{d}-B$, with positive integers $A, B$. This polynomial is of the required form for application of Theorem 5.2, whose conclusion contradicts $\liminf \#(y, N) N^{-1 / d}=0$.

So for example the number

$$
\left(\sum_{n \geq 0} \frac{1}{2^{n^{5}}}\right)^{4}
$$

is irrational; the number being 4-th-powered does not, in the sense of Corollary 5.3, have enough 1-bits.

Theorem 5.2 reveals that the assignments $y=\sqrt{2}$ or $y=(-1+\sqrt{5}) / 2$ (the golden mean) each have $\#(y, N)>(1-\epsilon) \sqrt{N}$ for large enough $N$; in the latter case one may use the polynomial equation $x^{2}+2 x-4=0$, whose root $-1+\sqrt{5}$ is in $(1,2)$. On a historical note: J. Samborski, in a published problem [34], asked for a proof that $\#(y, N)<5 \cdot 2^{N-2}$ —an interesting, hard bound but asymptotically very much weaker than our square-root density.

\section{Bounds on BBP tails}

Now we desire to lift all restrictions on the coefficient signs, except the high coefficient $A_{D}>0$ and contemplate the following representation relation (in this section we assume $x \in(1,2)$ is algebraic of degree $D>1$, see the remarks opening the proof of Theorem 5.2):

$$
A_{D} x^{D}+\cdots+A_{1} x+A_{0}=0=A_{0}+\sum_{n \geq 0} \frac{1}{2^{n}} \sum_{d=1}^{D} A_{d} r_{d}(x, n) .
$$

Consider a shift by $R$ bits of all entities, so that

$$
-2^{R} A_{0}=I(x, R)+T(x, R),
$$

where $I(x, R)$ is an integer and the BBP tail is defined

$$
T(x, R)=\sum_{m \geq 1} \frac{1}{2^{m}} \sum_{d=1}^{D} A_{d} r_{d}(x, R+m)=\sum_{d=1}^{D} A_{d} T_{d}(x, R),
$$


where as in Section 2 we identify a tail component

$$
T_{d}(x, R)=\sum_{m \geq 1} \frac{r_{d}(x, R+m)}{2^{m}} .
$$

The concept of BBP tail comes from the Bailey-Borwein-Plouffe formalism [2], whereby one may rapidly compute isolated bits of certain binary expansions - such as for $\pi, \log 2$ - by rapid computation of the integer $I(x, R)$ and rigorous control of the "tail" $T(x, R)$.

Remarkably, it is a fact that for the algebraic $x$ in question, $T(x, R)$ is always an integer, for the simple reason that $T(x, R)=-2^{R} A_{0}-I(x, R)$. To facilitate further analysis, we shall require a bound on the average absolute value of the tails $T(x, R)$ in terms of one value of $\#(x, N)$ :

Lemma 6.1. Let $x$ be an algebraic number in $(1,2)$ of degree $D>1$ with minimum integer polynomial $A_{D} x^{D}+A_{D-1} x^{D-1}+\cdots+A_{0}$, so $A_{D}>0$. Let $N \geq 2 D$ and set $K=\lceil 2 D \lg N\rceil$. Then for $1 \leq d \leq D$ we have

$$
\sum_{1 \leq R \leq N-K} T_{d}(x, R)<\#(x, N)^{d}+1,
$$

and so

$$
\sum_{1 \leq R \leq N-K}|T(x, R)|<\sum_{d=1}^{D}\left|A_{d}\right|\left(\#(x, N)^{d}+1\right) .
$$

Proof. We have

$$
\begin{aligned}
\sum_{R \leq N-K} T_{d}(x, R)= & \sum_{m \geq 1} 2^{-m} \sum_{R \leq N-K} r_{d}(x, R+m) \\
\leq & \sum_{m=1}^{K} 2^{-m} \sum_{R \leq N} r_{d}(x, R) \\
& +2^{-K} \sum_{m>K} 2^{K-m} \sum_{R \leq N-K} r_{d}(x, R+m) \\
< & \sum_{R \leq N} r_{d}(x, R)+2^{-K} \sum_{K \leq R \leq N} T_{d}(x, R) .
\end{aligned}
$$

Using (1) and Theorem 2.1 we have

$$
\sum_{1 \leq R \leq N-K} T_{d}(x, R) \leq \#(x, N)^{d}+N^{-2 D}(N+1)^{d},
$$

and the lemma is proved.

We shall use Lemma 6.1 to show that if $\#(x, N)$ is small, then not too many values of $T(x, R)$ are positive. Counter to this, the following lemma gives conditions on when there are many positive tails $T(x, R)$. 
Lemma 6.2. Let $x$ be an algebraic number in $(1,2)$ of degree $D>1$. Suppose that $R_{0}<R_{1}$ are positive integers with $r_{D-1}(x, R)=0$ for all integers $R \in\left(R_{0}, R_{1}\right]$ and $T\left(x, R_{1}\right)>0$. Then $T(x, R)>0$ for every integer $R \in\left[R_{0}, R_{1}\right]$.

Proof. Say the minimum integer polynomial for $x$ is $A_{D} x^{D}+A_{D-1} x^{D-1}+$ $\cdots+A_{0}$. As the 0-bit of $x$ is 1 it follows that $r_{d}(x, N) \geq r_{d-1}(x, N)$ for $d \geq 2$. Thus the hypothesis implies that for each $d=1,2, \ldots, D-1$ we have $r_{d}(x, R)=0$ for each integer $R \in\left(R_{0}, R_{1}\right]$. From the general recurrence relation on tails,

$$
T(x, R-1)=\frac{1}{2} T(x, R)+\frac{1}{2} \sum_{d=1}^{D} A_{d} r_{d}(x, R)=\frac{1}{2} T(x, R)+\frac{1}{2} A_{D} r_{D}(x, R) .
$$

Assuming inductively that $T(x, R)>0$, and using $A_{D}>0$, we get $T(x, R-$ 1) $>0$.

\section{The central theorem regarding general real algebraic numbers}

We have established that for a certain restricted class of algebraics $y$ of degree $D \geq 2$,

$$
\#(|y|, N)>(1-\epsilon)\left(1+\lg A_{D}\right)^{-1 / D} N^{1 / D}
$$

for sufficiently large $N$, where $A_{D}$ is the leading coefficient of the minimum integer polynomial for the normalized algebraic $x=|y| / 2^{\lfloor\lg |y|\rfloor}$. Now we move to general algebraics, so that there will be no coefficient constraints except for the natural $A_{D}>0$. Fortunately, we shall achieve a bound which is weaker only by an overall constant factor.

Theorem 7.1. For real algebraic $y$ of degree $D>1$ and for any $\epsilon>0$ we have for sufficiently large $N$ (with threshold depending on $y, \epsilon$ )

$$
\#(|y|, N)>(1-\epsilon)\left(2 A_{D}\right)^{-1 / D} N^{1 / D},
$$

where $A_{D}>0$ is the leading coefficient of the minimum integer polynomial of $x=|y| / 2^{\lfloor\lg |y|\rfloor}$.

Proof. As in the proof of Theorem 5.2, we use the normalized algebraic $x \in$ $(1,2)$, observing that $\#(x, N), \#(|y|, N)$ differ only by an integer constant, so again we may concentrate on the bit-counting for $x$. Suppose $\#(x, N) \leq$ $c N^{1 / D}$. Then from (1) applied for $d=D-1$, and the fact that each $r_{D-1}(x, R)$ is a nonnegative integer, we have that the number of integers $R \leq N$ with $r_{D-1}(x, R)>0$ is at most $c^{D-1} N^{1-1 / D}$. Say these $R$ 's are $0=R_{1}<R_{2}<\cdots<R_{M}$, where $M \leq c^{D-1} N^{1-1 / D}$. Let $R_{M+1}=N$. Trivially we have

$$
\sum_{i=1}^{M}\left(R_{i+1}-R_{i}\right)=N
$$


For $\delta>0$, let $I$ denote the set of numbers $i \leq M$ such that $R_{i+1}-R_{i} \geq$ $\frac{\delta}{3} c^{1-D} N^{1 / D}$. (Ultimately we transform $\delta$ into the $\epsilon$ of the theorem.) We have

$$
\sum_{i \in I}\left(R_{i+1}-R_{i}\right) \geq N-\frac{\delta}{3} c^{1-D} N^{1 / D} M \geq\left(1-\frac{\delta}{3}\right) N .
$$

Now we wish to show, if $i \in I$ and if integer $R \in\left(R_{i}, R_{i+1}-D \log N\right]$ has $r_{D}(x, R)>0$, then $T(x, R-1)>0$ :

$$
\begin{aligned}
T(x, R-1) & \geq \frac{1}{2} A_{D}-\sum_{d=1}^{D-1}\left|A_{d}\right| \sum_{m \geq 1} 2^{-m} r_{d}(x, R-1+m) \\
& =\frac{1}{2} A_{D}-\sum_{d=1}^{D-1}\left|A_{d}\right| \sum_{m>R_{i+1}-R} 2^{-m} r_{d}(x, R-1+m) \\
& =\frac{1}{2} A_{D}-\sum_{d=1}^{D-1}\left|A_{d}\right| 2^{R-R_{i+1}} T_{d}\left(x, R_{i+1}-1\right) \\
& \geq \frac{1}{2}-N^{-D} \sum_{d=1}^{D-1}\left|A_{d}\right|(N+d)^{d} /(d-1) ! N
\end{aligned}
$$

where this last inequality follows from Theorem 2.1. Thus, for sufficiently large $N$, the positivity of the tail $T(x, R-1)$ for such an $R$ is established. Now if $r_{1}(x, j)>0$ and $i \leq M$ then $r_{D}\left(x, R_{i}+j\right)>0$. A key observation now is: By the Thue-Siegel-Roth implication Theorem 3.1, for $N$ sufficiently large and for any $i \in I$, there is some integer $j_{i}$ with

$$
j_{i} \in\left(\frac{1}{2+\delta / 2}\left(R_{i+1}-R_{i}-D \log N\right), R_{i+1}-R_{i}-D \log N\right)
$$

and $r_{1}\left(x, j_{i}\right)>0$. We conclude that $r_{D}\left(x, R_{i}+j_{i}\right)>0$, so it follows from our previous reasoning that $T\left(x, R_{i}+j_{i}-1\right)>0$. Then from Lemma 6.2 we have $T(x, R)>0$ for every integer $R \in\left[R_{i}, R_{i}+j_{i}\right)$. Hence $T(x, R)>0$ for at least

$$
\frac{1}{2+\delta / 2} \sum_{i \in I}\left(R_{i+1}-R_{i}-D \log N\right)
$$

values of $R \leq N$. But this last expression is at least

$$
\frac{1}{2+\delta / 2}\left(1-\frac{\delta}{3}\right) N-D c^{D-1} N^{1-1 / D} \log N
$$

which is at least $\left(\frac{1}{2}-\frac{\delta}{3}\right) N$ for all sufficiently large values of $N$. 
We now show that if $c$ is too small, this last conclusion is impossible. By Lemma 6.1 we have (with $K$ as in the lemma)

$$
\begin{aligned}
\sum_{R \leq N-K}|T(x, R)| & \leq \sum_{d=1}^{D}\left|A_{d}\right|\left(\#(x, N)^{d}+1\right) \\
& \leq \sum_{d=1}^{D}\left|A_{d}\right|\left(c^{d} N^{d / D}+1\right) \\
& =A_{D} c^{D} N+O\left(N^{1-1 / D}\right) .
\end{aligned}
$$

Suppose now that $c \leq\left((2+\delta) A_{D}\right)^{-1 / D}$. It follows from this last calculation and the fact that each $T(x, R)$ is an integer that $T(x, R)>0$ for at most $\frac{1}{2+\delta} N+O\left(N^{1-1 / D}\right)$ values of $R \leq N$. So for $N$ sufficiently large, this assertion is incompatible with the assertion that $T(x, R)>0$ for at least $\left(\frac{1}{2}-\frac{\delta}{3}\right) N$ values of $R \leq N$. Finally, for the arbitrary positive $\delta$ we set $\epsilon=1-(1+\delta / 2)^{-1 / D}$ to obtain the statement of the theorem.

\section{Implications of the central theorem}

Theorem 7.1 can be used to establish transcendency of a class of binary expansions, as in

Theorem 8.1. Let a function $f: \mathcal{R} \rightarrow \mathcal{R}$ be strictly monotonic increasing, with $f$ attaining integer values for integer arguments. If for any $\epsilon>0$ the inverse of $f$ satisfies

$$
f^{-1}(y)=O\left(y^{\epsilon}\right)
$$

then the number

$$
x=\sum_{n \geq 0} \frac{1}{2^{f(n)}}
$$

is transcendental.

Proof. Note that the bit positions $f(n)$ are distinct, so the observation

$$
f^{-1}(N)=\#\{n>0: f(n) \leq N\}=\#(x, N)
$$

means $\#(x, N)=O\left(N^{\epsilon}\right)$, which for algebraic $x$ is incompatible with Theorem 7.1.

Corollary 8.2. For any real $\alpha>1$ the number

$$
m_{\alpha}=\sum_{n \geq 0} \frac{1}{2^{\left\lfloor\alpha^{n}\right\rfloor}}
$$


is transcendental. So the Kempner-Mahler number $M=m_{2}$ and the Fibonacci binary involving the Fibonacci numbers $\left(F_{n}\right)=(0,1,1,2,3,5, \ldots)$

$$
X=\sum_{n \geq 0} \frac{1}{2^{F_{n}}}
$$

are transcendental. Finally, there are transcendental numbers of still greater 1-bit densities, such as

$$
Y=\sum_{n \geq 3} \frac{1}{2^{\left\lfloor n^{\log \log n}\right.}}
$$

Remark. Recall that the Thue-Siegel-Roth implication Theorem 3.1 handles $\alpha>2$.

Proof. As for $m_{\alpha}$, take $n_{0}=\lceil-\log (\alpha-1) / \log \alpha\rceil$ so that there is a strictly monotone function whose ineteger evaluations are $f(n)=\left\lceil\alpha^{n+n_{0}}\right\rceil$, with $f^{-1}(N)=O(\log N)$, so that Theorem 8.1 applies and the partial binary sum for $m_{\alpha}$ starting from index $n_{0}$, hence $m_{\alpha}$ itself, is transcendental. As for the Fibonacci binary, the $n$-th Fibonacci number can be written $\left.f(n)=\left((1+\tau)^{n}-(-\tau)^{n}\right)\right) / \sqrt{5}$, where $\tau=(\sqrt{5}-1) / 2$, so the growth of 1 's positions is essentially that of $m_{1+\tau}$ and again incompatible with Theorem 7.1 if $X$ is assumed algebraic. For the number $Y$ it is evident that $\#(Y, N) \sim N^{1 / \log \log N}$ which is of slower growth than any positive power of $N$.

We can also use Theorem 7.1 to generate results on algebraic degrees for certain constants, as in the following (as before let us stipulate that the algebraic degree of a transcendental is $\infty$ ):

Theorem 8.3. For positive integer $k$ the number

$$
X_{k}=\sum_{n \geq 0} \frac{1}{2^{n^{k}}}
$$

has algebraic degree at least $k$, while the number

$$
P_{k}=\sum_{p \text { prime }} \frac{1}{2^{p^{k}}}
$$

has algebraic degree at least $k+1$.

Proof. In the first case, $\#\left(X_{k}, N\right)=\#\left\{n^{k} \leq N\right\}<C N^{1 / k}$, so by Theorem 7.1 we must have degree $D \geq k$. In the second case we have $\#\left(P_{k}, N\right)=$ $\#\left(p^{k} \leq N\right)=\pi\left(N^{1 / k}\right)<A N^{1 / k} /(\log N)$ for a constant $A$, so that again by Theorem 7.1, we must have $D>k$.

Thus for example neither $P_{2}$ nor $X_{3}$ is a quadratic irrational. The case of $X_{2}$ is just the previously mentioned number $z=\sum 1 / 2^{n^{2}}$, on which number we focus attention in the next section. 


\section{Study of a "borderline" number}

The number

$$
z=\sum_{n \geq 0} \frac{1}{2^{n^{2}}}=\frac{1}{2}\left(\theta_{3}\left(\frac{1}{2}\right)+1\right)
$$

is, with respect to the present treatment, a "borderline" case because, as we have seen, a square-root density of 1's is beyond reach of our methods. Recall also as in Section 4 that there are numbers with the same essential density of 1's as $z$ but for which products of such numbers can be rational. Note that

$$
z^{\prime}=2 z-1=\theta_{3}\left(\frac{1}{2}\right)
$$

so that

$$
z^{\prime 2}=\sum_{n \geq 0} \frac{r_{2}(n)}{2^{n}},
$$

where now we are using the standard notation of $r_{2}(n)$ for the number of representations $n=a^{2}+b^{2}$ for $a, b \in \mathbf{Z}$, counting sign and order. It will be convenient therefore to study $z^{\prime}$, from which algebraic properties of $z$ follow. Incidentally $z^{\prime 2}$ has some interesting numerological features; for one thing it is very close to $\pi / \log 2$; in fact the approximation

$$
z^{\prime 2} \approx \frac{\pi}{\log 2}\left(1+2 e^{-\pi^{2} / \log 2}\right)^{2}=4.53237201425897410082795 \ldots
$$

can be obtained via Jacobi $\theta$-transformation, and remarkably is correct to the implied 23 decimal places in the above display. It is fascinating that such relations between $z^{\prime 2}$ and fundamental constants exist even though, as we shall prove, almost all of the binary bits of $z^{\prime 2}$ are 0 's.

It is one of the earliest results in additive number theory, due to Jacobi, that

$$
r_{2}(n)=4 \sum_{d \mid n, d \text { odd }}(-1)^{(d-1) / 2} .
$$

It turns out that the representation count $r_{2}(n)$ is positive if and only if every prime $p \equiv 3(\bmod 4)$ dividing $n$ does so to an even power. Thus, the representation sequence $\left(r_{2}(0), \ldots\right)$ has zeros in any position $n=3 k$ with $(3, k)$ coprime, and so on. Deeper results on $r_{2}$ include the fact that the number of representable integers not exceeding $N$ behaves according to the Landau theorem:

$$
\sum_{n \leq N} H\left(r_{2}(n)\right) \sim L \frac{N}{\sqrt{\log N}}
$$


where the Landau constant is

$$
L=\left(\frac{1}{2} \prod_{p \equiv 3(\bmod 4)}\left(1-\frac{1}{p^{2}}\right)^{-1}\right)^{1 / 2}=0.764223653 \ldots
$$

(See [15] for descriptions of this and other facets of sums of squares.) The Landau density of representable numbers does not on the face of it imply a similar density of 1-bits in the expansion of $z^{\prime 2}$.

Evidently we have

$$
z^{2}=4 \sum_{d \text { odd }} \frac{(-1)^{(d-1) / 2}}{2^{d}-1}
$$

This form is reminiscent of the Erdős-Borwein number

$$
E=\sum_{n>0} \frac{1}{2^{n}-1}=\sum_{m>0} \frac{d(m)}{2^{m}},
$$

where $d(m)$ denotes the number of divisors of $m$. The constant $E$ was proven irrational by Erdős [13] who used number-theoretical arguments (outlined in [4]) which did, in fact, motivate our present analysis of ${z^{\prime}}^{2}$. Later the irrationality of such forms was established via Padé approximants, by P. Borwein [8].

What we shall show is that $z^{\prime}$ is not a quadratic irrational, and so neither is $z$. In one sense this is stronger than the quoted irrationality results for the number $E$. On the other hand, it is already known that theta functions at algebraic arguments, hence $z, z^{\prime}$, are transcendental $[5,12]$. To effect our nonquadratic-irrationality proof, we shall follow the same basic prescription as for Theorem 7.1; namely, we establish upper bounds on the size of representations, and employ some knowledge of zero-runs. As for upper bounds, it is known [17] that for any fixed $\epsilon>0$ we have

$$
r_{2}(n)<2^{\left(\frac{1}{2}+\epsilon\right) \frac{\log n}{\log \log n}}
$$

for sufficiently large $n$. Note that this bound is much tighter than the general one of Theorem 2.1. This tighter bound works well with what we can show about zero-runs:

Theorem 9.1. Let $\epsilon>0$ be arbitrary, but fixed, and define

$$
u_{\epsilon}(x)=\frac{1-\epsilon}{2 L} \frac{\log x}{\sqrt{\log \log x}}
$$

where $L$ is the Landau constant. Then for sufficiently large $x$ there is a square integer $M$ with $M<x$ and an integer $a<M$ such that $r_{2}(n)=0$ whenever

$$
n \equiv a+i(\bmod M), \quad 1 \leq i \leq u_{\epsilon}(x) .
$$


Proof. Let $x$ be large and let

$$
u=\left\lfloor\frac{1-\epsilon}{2 L} \frac{\log x}{\sqrt{\log \log x}}\right\rfloor .
$$

Let $v_{p}(n)$ denote the exponent on the prime $p$ in the prime factorization of $n$. Cast out from $[1, u]$ any integer $o$ with $v_{p}(n)$ odd for some prime $p<u / \log u, p \equiv 3(\bmod 4)$. Let $\mathcal{E}$ denote the set of remaining numbers $n$, and let $E$ denote the cardinality of $\mathcal{E}$. Also, let $\mathcal{E}_{1}$ denote the number of integers in $[1, u]$ which are the sum of two squares, and let $E_{1}$ denote the cardinality of $\mathcal{E}_{1}$. By the Landau theorem,

$$
E_{1} \sim \frac{L u}{\sqrt{\log u}} .
$$

Clearly, $\mathcal{E}_{1} \subset \mathcal{E}$. In particular, $E-E_{1}$ is at most the number of integers $n \in$ $[1, u]$ divisible by some prime $p$ with $u / \log u \leq p \leq u$ and $p \equiv 3(\bmod 4)$. Then

$$
E-E_{1} \leq u \sum_{u / \log u \leq p \leq u} \frac{1}{p}=O(u \log \log u / \log u) .
$$

We conclude that

$$
E \sim \frac{L u}{\sqrt{\log u}} .
$$

Label the members of $\mathcal{E}$ as $n_{1}, n_{2}, \ldots, n_{E}$.

Next, let $M_{1}=\prod p^{a_{p}}$, where $p$ runs over the primes with $p \equiv 3(\bmod 4)$, $p<u / \log u$, and $a_{p}=2\lceil(\log u) /(2 \log p)\rceil$. (Thus, $a_{p}$ is the least even integer with $p^{a_{p}} \geq u$.) We have $\log M_{1}=O(u / \log u)$.

Let

$$
v=\left\lfloor\frac{\log x}{1+\epsilon}\right\rfloor,
$$

and let $M_{2}=\prod p^{2}$ where $p$ runs over the primes $p \equiv 3(\bmod 4)$ with $u / \log u \leq p \leq v$. Then $\log M_{2} \sim v$, so that for $x$ sufficiently large we have $M:=M_{1} M_{2}<x$. Label the prime factors of $M_{2}$ as $p_{1}, p_{2}, \ldots, p_{F}$, where $F \sim v /(2 \log v)$. We have

$$
\begin{gathered}
E \sim \frac{L u}{\sqrt{\log u}} \sim \frac{1-\epsilon}{2} \frac{\log x}{\log \log x}, \\
F \sim \frac{v}{2 \log v} \sim \frac{1}{2(1+\epsilon)} \frac{\log x}{\log \log x},
\end{gathered}
$$

so that for $x$ sufficiently large we have $F \geq E$.

For $1 \leq i \leq E$ let $r_{i}$ be a solution to

$$
n_{i}+r_{i} M_{1} \equiv p_{i}\left(\bmod p_{i}^{2}\right) .
$$

Further, let the integer $r$ satisfy

$$
r \equiv r_{i}\left(\bmod p_{i}^{2}\right), \quad \text { for } 1 \leq i \leq E .
$$


Let $h$ be an arbitrary integer. If $n$ is an integer in $[1, u]$ that is not in $\mathcal{E}$, then $v_{p}\left(n+r M_{1}+h M\right)$ is odd for some prime $p \mid M_{1}$ and so $r_{2}(n)=0$. Suppose $n=n_{i} \in \mathcal{E}$. Then $v_{p_{i}}\left(n+r M_{1}+h M\right)=1$, and so $r_{2}(n)=0$. Thus, with $a=r M_{1}$ we have that $r_{2}(a+i+h M)=0$ for $1 \leq i \leq u$. This completes the qroof of the theorem.

Corollary 9.2. For integer $n$ sufficiently large, the interval $\left(n^{2}, n^{2}+n\right)$ contains a zero-run of the $r_{2}$ representation of length at least $u_{\epsilon}(n)$.

Proof. Take $x=n / 3$ in Theorem 9.1. Then for relevant $M$ and $a$, the position

$$
n^{2}+\left(a+1+M-\left(n^{2} \bmod M\right)\right) \leq n^{2}+2 n / 3
$$

is the start of a zero-run of length $u_{\epsilon}(n / 3) \sim u_{\epsilon}(n)$, which run for sufficiently large $n$ is contained in $\left(n^{2}, n^{2}+n\right)$.

We are now in a position to use representation bounds and the zero-run bound of Theorem 9.1, to establish

Theorem 9.3. The number $z=\sum_{n \geq 0} 1 / 2^{n^{2}}$ is not a quadratic irrational.

Proof. We shall focus on the number $z^{\prime}=\sum_{n \in \mathbf{Z}} 1 / 2^{n^{2}}$ whence the result will follow for $z$. Assume that

$$
A_{2} z^{\prime 2}+A_{1} z^{\prime}+A_{0}=0
$$

Consider the interval $\left[n^{4},\left(n^{2}+1\right)^{2}\right]$ and within that, positions

$$
n^{4}, n^{4}+f, n^{4}+f+Z, n^{4}+n^{2},\left(n^{2}+1\right)^{2} .
$$

By Corollary 9.2, for sufficiently large $n$, these positions are in order, with a zero-run length $Z=\left\lfloor u_{\epsilon}\left(n^{2}\right)\right\rfloor$, so that $\left(r_{2}\left(n^{4}+f+1\right), \ldots, r_{2}\left(n^{4}+f+Z\right)\right)$ is a length- $Z$ zero-vector. Note also that $r_{1}\left(n^{4}\right)=r_{1}\left(\left(n^{2}+1\right)^{2}\right)=1$, yet every other $r_{1}$ in the entire interval is zero. Thus

$$
T\left(z^{\prime}, n^{4}+n^{2}-1\right) \geq \frac{1}{2}-\frac{\left|A_{1}\right|}{2^{n^{2}}}>0 .
$$

Thus any tail $T\left(z^{\prime}, n^{4}\right), \ldots, T\left(z^{\prime}, n^{4}+n^{2}-1\right)$ is positive. However, using the upper bound on $r_{2}(n)$ to bound the tail component $T_{2}\left(z^{\prime}, n^{4}+f\right)$, we get

$$
T\left(z^{\prime}, n^{4}+f\right) \leq \frac{2 A_{2}}{2^{Z}} 2^{(2+4 \epsilon) \log n / \log \log n}+\frac{\left|A_{1}\right|}{2^{2 n^{2}-f}} .
$$

Since $Z$ has the $\sqrt{\log \log n}$ denominator, we have for sufficiently large $n$

$$
0<T\left(z^{\prime}, n^{4}+f\right)<1,
$$

a contradiction.

We now state the following result, which was first suggested to us by numerical computation. 
Theorem 9.4. Almost all bits of $z^{2}$ are 0's; in fact the 1's-count has asymptotic behavior

$$
\#\left(z^{2}, N\right) \sim C_{0} \frac{N}{\sqrt{\log N}},
$$

for an absolute constant $C_{0} \approx 0.7996 \ldots$ (we give a formula for $C_{0}$ in the proof).

Clearly, Theorem 9.4 implies that $z^{2}$ is irrational (because of arbitrarily long zero-runs), and Theorem 9.3 may well follow also from the asymptotic 1's density (although see Section 11). Incidentally the asymptotic density also holds for $z^{\prime 2}$, as follows from a slight modification (actually simplification) of the proof. In spite of the paucity of 1's for these squared numbers, higher powers such as $z^{3}, z^{4}$ are likely 2-normal. Indeed, all such higher powers will involve interfering carries. For example, it is known that $r_{3}(n)>0$ for a limiting fraction $5 / 6$ of all $n$ (see [15]), so the carry problem for $z^{3}$ is already formidable.

The proof of Theorem 9.4 is based on the following two lemmas.

Lemma 9.5. There is an absolute constant $c$ such that for any integers $N, B \geq 2$, the number of integers $n \leq N$ with $r_{2}(z, n)>0$ and $r_{2}(z, m)>0$ for some integer $m$ with $0<|n-m|<B$ is at most $c B N / \log N$.

Lemma 9.6. For any positive integers $B, N$, the number of integers $n \leq N$ with $r_{2}(z, n) \geq B$ is at most $(\sqrt{N}+1)^{2} / B$.

Note that Lemma 9.6 is very easy. The assertion follows instantly from the inequality $\sum_{n \leq N} r_{2}(z, n) \leq(\sqrt{N}+1)^{2}$. We postpone the proof of Lemma 9.5 until later. First we see how Theorem 9.4 follows from the lemmas.

Proof. (Theorem 9.4.) Let $b(m)=\#(m, 0)$ denote the number of 1 's in the binary representation of the nonnegative integer $m$, and let $b(0)=0$. It follows from Theorem 4.2 and the fact that $r_{2}(z, n) \leq n^{o(1)}$ that for $N$ large,

$$
\#\left(z^{2}, N\right) \leq \sum_{n \leq N+\log N} b\left(r_{2}(z, n)\right) .
$$

The goal is to get a similar-looking lower bound. Let $S_{N}$ denote the set of natural numbers $n \leq N$ such that $n$ is not a square and

$$
\begin{aligned}
r_{2}(z, n) & >0, \\
r_{2}(z, m) & =0 \text { for } 0<|n-m|<3 \lg \lg N, \\
r_{2}(z, m) & <(\lg N)^{2} \text { for }|n-m|<2 \lg N .
\end{aligned}
$$


Note that if $n \in S_{N}$ and $N$ is sufficiently large then

$$
\sum_{m>n} \frac{r_{2}(z, m)}{2^{m}}<\frac{1}{2^{n}}
$$

Indeed, we first note that

$$
\sum_{m \geq n+2 \lg N} \frac{r_{2}(z, m)}{2^{m}}<\sum_{m \geq n+2 \lg N} \frac{m}{2^{m}}=O\left(\frac{1}{N 2^{n}}\right) .
$$

Next note that

$$
\begin{aligned}
\sum_{n+2 \lg N>m>n} \frac{r_{2}(z, m)}{2^{m}} & =\sum_{n+2 \lg N>m \geq n+3 \lg \lg N} \frac{r_{2}(z, m)}{2^{m}} \\
& \leq \sum_{n+2 \lg N>m \geq n+3 \lg \lg N} \frac{(\lg N)^{2}}{2^{m}} \\
& =O\left(\frac{1}{2^{n} \lg N}\right) .
\end{aligned}
$$

Thus, we have (4). Further, for $n \in S_{N}$ and $N$ large we have

$$
\sum_{m \geq n} \frac{r_{2}(z, m)}{2^{m}}<\frac{r_{2}(z, n)+1}{2^{n}}<\frac{(\lg N)^{2}+1}{2^{n}}<\frac{1}{2^{n^{\prime}}},
$$

where $n^{\prime}<n$ is the largest number with $r_{2}\left(z, n^{\prime}\right)>0$. We conclude from these estimates that appearing in the bit stream for $z^{2}$ we see intact all of the bits of the numbers $r_{2}(z, n)$ for $n \in S_{N}$, when $N$ is large. Thus, we have for large $N$ that

$$
\#\left(z^{2}, N\right) \geq \sum_{n \in S_{N}} b\left(r_{2}(z, n)\right) .
$$

It follows from the lemmas that the number of integers $n \leq N$ with $r_{2}(z, n)>0$ that are not in $S_{N}$ is $O(N \log \log N / \log N)$. The number of 1-bits contributed to \# $\left(z^{2}, N\right)$ from $n \leq N$ with $n \notin S_{N}$ and $r_{2}(z, n)<$ $2^{(\log N)^{1 / 4}}$ is at most

$$
O\left((\log N)^{1 / 4} \frac{N \log \log N}{\log N}\right)=o\left(\frac{N}{\sqrt{\log N}}\right) .
$$

And, by Lemma 9.6 there are at most $O\left(N / 2^{(\log N)^{1 / 4}}\right)$ values of $n \leq N$ with $r_{2}(z, n)>2^{(\log N)^{1 / 4}}$. Since $b\left(r_{2}(z, n)\right)=o(\log n)$, the contribution of these values of $n$ to $\#\left(z^{2}, N\right)$ is also $o(N / \sqrt{\log N})$. It follows that

$$
\#\left(z^{2}, N\right)=\sum_{n \leq N} b\left(r_{2}(z, n)\right)+o(N / \sqrt{\log N}) .
$$


Using the identity $z^{\prime 2}=4 z^{2}-4 z+1$ and that $r_{2}(n)=4 r_{2}(z, n)$ when $n$ is not a square, and $r_{2}(n)=4 r_{2}(z, n)-4 \geq 0$ when $n$ is a positive square, we further see that

$$
\#\left(z^{2}, N\right)=\sum_{n \leq N} b\left(r_{2}(n)\right)+o(N / \sqrt{\log N}) .
$$

Hence it is sufficient to estimate this last sum.

Suppose $n=n_{1} n_{2} n_{3}$ where $n_{i}$ is the largest divisor of $n$ composed of primes that are congruent to $i(\bmod 4)$. We have $r_{2}(n)>0$ if and only if $n_{3}$ is a square. And if $n_{3}$ is a square, then $r_{2}(n) / 4=d\left(n_{1}\right)$, where $d$ is the standard divisor function. It follows that if $n_{3}$ is a square and if $g(n)$ denotes the largest squarefull divisor of $n_{1}$ then $r_{2}(n) / d(g(n))$ is a power of 2 , so that

$$
b\left(r_{2}(n)\right)=b(d(g(n))) .
$$

Incidentally by squarefull is meant an integer none of whose prime factors appears to the power 1 .

We now count the number $T_{g}(N)$ of integers $n \leq N$ with $r_{2}(n)>0$ and such that $g(n)=g$, where $g$ is a given squarefull integer all of whose primes are congruent to $1(\bmod 4)$. It is not too difficult to see that

$$
T_{g}(N) \sim L \frac{N}{\sqrt{\log N}} \frac{\alpha}{g} \prod_{p \mid g}\left(1-\frac{1}{p}\right)\left(1-\frac{1}{p^{2}}\right)^{-1},
$$

where

$$
\alpha=\prod_{p \equiv 1 \bmod 4}\left(1-\frac{1}{p^{2}}\right)=\frac{16 L^{2}}{\pi^{2}},
$$

and where $p$ in these formulae runs over primes. Letting $\psi(g)=g \prod_{p \mid g}(1+$ $1 / p)$, we thus have that

$$
T_{g}(N) \sim \frac{16 L^{3}}{\pi^{2}} \frac{N}{\psi(g) \sqrt{\log N}} .
$$

Hence, we have Theorem 9.4 with

$$
C_{0}=\frac{16 L^{3}}{\pi^{2}} \sum_{g} \frac{b(d(g))}{\psi(g)},
$$

where $g$ runs over the squarefull integers divisible solely by primes that are congruent to $1(\bmod 4)$. Note that this sum is convergent, which convergence partially justifies the adding of the asymptotic relations for $T_{g}(N)$.

We do not give a proof of the asymptotic relations for $T_{g}(N)$, but these can be achieved as corollaries of the Landau asymptotic formula. In Section 
10 we give numerical verification of Theorem 9.4. We close the present section with a proof of Lemma 9.5.

Proof. (Lemma 9.5.) Let $r^{\prime}(n)$ denote the number of coprime representations of $n$ as the sum of two squares. First we count the number of integers $n \leq N$ for which $r^{\prime}(n)>0$ and for which $r^{\prime}(m)>0$ for some integer $m$ with $0<|n-m|<B$. Note that for $r^{\prime}(m)$ to be positive it is necessary that $m$ is not divisible by any prime congruent to $3(\bmod 4)$, that is, that $m_{3}=1$. (This condition is almost sufficient: to make it sufficient it should also be the case that $m_{2}$, the 2-power in $m$, is not a power of 4.) For a given integer $k>0$, the number of integers $n \leq N$ with both $r^{\prime}(n)>0, r^{\prime}(n+k)>0$ is, by Theorem 2.3 in [16], at most

$$
c^{\prime} \psi(k) \frac{N}{\log N},
$$

where $c^{\prime}$ is an absolute constant and where $\psi$ is defined in the proof of Theorem 9.4. (Actually one can have the smaller factor $\psi\left(d_{3}\right)$, but this is unimportant.) Since

$$
\sum_{k \leq B} \psi(k)=O(B)
$$

as is easily seen by elementary methods (see [17], Ch. 18), it follows that the number of $n \leq N$ with $r^{\prime}(n)>0, r^{\prime}(n+k)>0$ for some integer $k$ with $0<|k|<B$ is $O(B N / \log N)$. This proves the lemma for the function $r^{\prime}$. To get it for $r_{2}(z, n)$ we generalize the above proof for the case $u^{2}\left|n, v^{2}\right| n+k$, where $u v$ is divisible only by primes that are congruent to $3(\bmod 4)$ and where $r^{\prime}\left(n / u^{2}\right)>0, r^{\prime}\left((n+k) / v^{2}\right)>0$. For any fixed choice for $u, v$ we get an estimate of $O\left(\psi(k) N /\left(u^{2} v^{2} \log N\right)\right)$ for the number of such $n \leq N$. Now we sum over $k, u, v$ getting the lemma.

\section{Numerical experiments for $C_{0}$}

The intricacies of the borderline number $z$ and its powers show that global bit-density arguments alone are insufficient to handle low 1's-density cases: We required number theory to focus on certain details of the bit pattern. Later in the research, we found that computational aspects - such as bit-counting - for $z^{2}$ are nontrivial. In attempts to verify Theorem 9.4 empirically - in particular, to justify the value of $C_{0}$ - the present authors were met with considerable computational consternation. There are two basic difficulties that need be overcome. Note that calculation of $C_{0}$ from the sum formula is not too hard, and gives us the cited $0.7996 \ldots$ value that we obtained by summing over squarefull $g \leq 10^{5}, 10^{6}, 10^{7}$ in succession, then extrapolating on the assumption of a reasonable form for the series-truncation error. The remaining difficulties all pertain to the actual counting of representations up through some large $n$. 
The first difficulty is that the Landau asymptotic formula is, for all practical purposes, generally below the mark, in the sense that a more accurate formula, also due to Landau, is [23]

$$
\sum_{n \leq N} H\left(r_{2}(n)\right)=L \frac{N}{\sqrt{\log N}}\left(1+\frac{C_{1}}{\log N}\right)+o\left(\frac{N}{(\log N)^{3 / 2}}\right),
$$

where $C_{1}=0.5819 \ldots$ is yet another constant. One might also use the Ramanujan form [18]

$$
\sum_{n \leq N} H\left(r_{2}(n)\right) \sim L \int_{0}^{N} \frac{d x}{\sqrt{\log x}}
$$

which is reminscent of the logarithmic integral $\operatorname{Li}(x)$ which, as is well known, stands as a better approximation to $\pi(x)$ than the classic $x / \log x$. Remarkably, for the original Landau expression $L N / \sqrt{\log N}$ to be accurate to say 1 per cent of an empirical count of representables, one has to go up to about $N=e^{50} \approx 10^{22}$, or about a mole of bits. That is more than the digital storage presently available on the entire planet.

The second computational difficulty is that the proof of Theorem 9.4 basically tells us that most $r_{2}$ values eventually "separate" so that carries do not interfere. When does separation become significant? A very rough heuristic runs as follows. For very large $N$ the mean separation between positive representation counts is about $\sqrt{\log N} / L$, and this should be greater than the base-2 logarithm of the largest $r_{2}$ values of the region. So, and again this is quite heuristic, for significant separation we should have

$$
\frac{\sqrt{\log N}}{\log \log N} \approx \frac{2}{L}
$$

which leads to the estimate of $N \approx 10^{80}$ bits, which is an oft-quoted estimate on the number of protons in the visible universe.

So these difficulties required the authors to calculate entities that converge to reasonable values for $N$ well below the aforementioned astronomical thresholds. It turns out that the quantity

$$
C(N)=L \frac{\sum_{n \leq N} b\left(r_{2}(n)\right)}{\sum_{n \leq N} H\left(r_{2}(n)\right)} \sim C_{0}
$$

is relatively well-behaved, and gives an excellent empirical value for $C_{0}$ (although, still, $N$ has to be taken painfully far and extrapolation techniques were required when our machinery reached its limit, as described below). Notice that this quantity $C(N)$ essentially measures - up to the $L$ factor - the number of bits per positive representation. The numerical results reported below suggest (and Theorem 9.4 implies) an amusing principle: The average number of bits in a positive representation count $r_{2}(n)$ 
is about $C_{0} / L=1.05$. That is, it is a very good bet that a random positive $r_{2}$ value is a power of 2 .

In order to calculate entities for $n$ up to and beyond say $10^{8}$, we employed a sieving expedient described in [11] for rapidly obtaining long strings of representation counts. The algorithm is quite simple:

(1) Create $N+1$ bins, say $B[0], \ldots, B[N]$ intended to hold representation counts,

then set $B[0]=1$ and zero all other bins.

(2) for (odd $d \leq N)\{$

if $(d \equiv 1 \bmod 4)$ add 4 to every $B[k d]$ with $k d \leq N$;

else subtract 4 from every $B[k d]$ with $k d \leq N$

\}

The result of this algorithm is that $r_{2}(n)$ is sitting in bin $B[n]$ for every $n \in[0, N]$. In the following table we denote by $\sum r_{2}$ the sum of counts $r_{2}(n)$ through $n=N$, by $\sum H$ the count of representables, by $c_{2}$ the number of $r_{2}(n)$ being a power-of-two, and by $\sum b$ the sum of all bit counts $b\left(r_{2}(n)\right)$ :

$\begin{array}{rrrrrrrr}N & L N / \sqrt{\log N} & \sum H & \sum r_{2} & c_{2} & \sum b & \#\left(z^{2}, N\right) & C(N) \\ 10^{6} & 205605.6 & 216342 & 3141552 & 204082 & 228646 & 213480 & 0.807683 \\ 10^{7} & 1903573.9 & 1985460 & 31416028 & 1877532 & 2093748 & 1968680 & 0.805901 \\ 10^{8} & 17805966.8 & 18457848 & 314159056 & 17482500 & 19436147 & 18353248 & 0.804725\end{array}$

Note the following interesting features of this table. The 1st-order Landau estimate (2nd column) indeed lags behind the representation count $\sum H$. Next, the similarity of $\sum r_{2}$ and the decimal digits of $\pi$ is, of course, not a coincidence: The celebrated Gauss circle problem starts with the proven estimate $\sum r_{2}=\pi N+o(N)$, whence one focuses attention on the little- $O$ term. Thus the present sieve technique for counting representations might be useful in numerical studies of the circle error. We see that indeed all but a few per cent of representables are a power-of-two (i.e., $c_{2}$ is close to $\left.\sum H\right)$. We stress that the $\#\left(z^{2}, N\right)$ column comes from processing of the $r_{2}$ with carry. Actually, rather than work out the carry chain for the $B[]$ bins, we instead obtained the \# column by simply squaring high-precision $z$ values. For both sheer arithmetic of that sort, or for our divisor-sieve, our machinery was not able to go up to $N=10^{9}$. Incidentally this is not because of CPU power - the sieve is quite fast, as are convolution methods of squaring reals - the problem is memory. Thus we are forced to extrapolate from the last column of data. Under Romberg extrapolation and the assumption of exponential approach, we estimate the final column's limit to be about 0.802 . This extrapolation is within 0.3 per cent of the theoretical $C_{0}=0.7996 \ldots$ and so we deem this numerical exercise successful. We should also mention that the $C(N)$ results, when other $N$ values are 
included in a larger table, are remarkably smooth, which in itself suggests the validity of extrapolation.

\section{Open problems}

Finally, we state some open problems:

- Is there a quantifiable sense in which the binary representation of $\sqrt{2}$ is not truly random? That is, we observe using the present techniques, that the representation list $\left(r_{2}(\sqrt{2}, 0), \ldots, r_{2}(\sqrt{2}, N)\right)$ for large $N$ cannot have any zero-run of more than $2 \lg N$ consecutive zeros. Evidently this is a hard constraint that one would not want to put on the representation sequence for a "truly random" bit generator.

- As for the Fibonacci binary $X$ of Corollary 8.2, what is the 1's density of $X^{2}$ ?

- What can be said about Fourier representations and bit densities? For example, for $x=\sqrt{2}$ the simple fact of $x^{2}=2$ can be recast as

$$
2=\int_{0}^{1}\left(\sum_{p \in \mathcal{P}(x)} \frac{e^{2 \pi i p t}}{2^{\lambda p}}\right)^{2} \frac{d t}{1-2^{\lambda-1} e^{-2 \pi i t}},
$$

where $\lambda \in(0,1)$ is a free parameter, and in principle such an integral representation should convey some information about the 1 's positions $p$ in the expansion of $\sqrt{2}$.

- For bases $b>2$ there is the difficulty of having more than two possible digits. What kinds of bounds might be placed on counts of 1's and 2's for ternary expansions of algebraic numbers?

- We have mentioned that the nonquadratic-irrationality Theorem 9.3 may well follow from the density Theorem 9.4. But there is an impasse which would have to be overcome. Namely, it turns out that, whereas $\#(z, N) \sim \sqrt{N}$, there exist reals $y$ with much greater than the square-root 1's density but such that still we have $\#(z+y, N) \sim$ $\sqrt{N}$. That is, adding $y$ to $z$ does not improve the 1 's count. To see this, define

$$
y=\sum_{n>1} \frac{2^{k_{n}}-1}{2^{n^{2}}}
$$

with arbitrary positive integers $k_{n}$ except for the constraint $k_{n}<$ $2 n-1$. When $z$ is added to such a $y$, the sets of $k_{n} 1$ 's are each obliterated by carry. This is an impasse because one cannot just infer the 1's density of $A z^{2}+B z$ merely by observing that the (rather high) density in Theorem 9.4 dominates the $\sqrt{N}$ density.

- The $C_{0}$ constant of Theorem 9.4 we have estimated as $0.7996 \ldots$ A fast algorithm for calculating the Landau constant $L$ itself is given 
in [14]. (In doing so, they effectively solved research Problem 1.88 of [10], which asks for a fast method.) Might there be a similar, fast construction for $C_{0}$ ?

- In recent times has emerged the field of "experimental mathematics," wherein one uses high-precision numerical relations such as linear reduction to suggest exact algebraic identities, in this way igniting a profusion of new results and theorems. One might say that the results of the present paper amount to a kind of "digitally motivated analysis" (we might abbreviate DMA), in which computers were not used (except to check various claims), yet results in the analysis field are obtained by thinking digitally, in our case thinking in binary. (And, we acknowledge the historical foreshadowing of "DMA", as in $[24,20,34,25]$.) A question, then, would be: What other aspects of analysis apart from transcendency might succumb to the "DMA" approach?

\section{Acknowledgments}

We are grateful to our colleagues J. Buhler, G. Fee, S.Wagon, and S. Wolfram for aid in this research. We owe special debts to J. Shallit and A. J. van der Poorten who provided us with historical perspective. We would also like to thank a referee who provided valuable comments and alerted us to several important references.

\section{References}

[1] J.-P. Allouche and J. Shallit, Automatic Sequences; Theory, Applications, Generalizations, Cambridge University Press, 2003.

[2] David H. Bailey, Peter B. Borwein and Simon Plouffe, "On The Rapid Computation of Various Polylogarithmic Constants," Mathematics of Computation, vol. 66, no. 218 (1997), pp. 903-913.

[3] David H. Bailey and Richard E. Crandall, "On the Random Character of Fundamental Constant Expansions," Experimental Mathematics, vol. 10 (2001), pp. 175-190.

[4] David H. Bailey and Richard E. Crandall, "Random generators and normal numbers," Experimental Mathematics, to appear.

[5] D. Bertrand, "Theta functions and transcendence," (Ramanujan Journal vol. 1 (1997), pp. 339-350.

[6] É. Borel, "Sur les chiffres décimaux de $\sqrt{2}$ et divers problèmes de probabilités en chaine," C. R. Acad. Sci. Paris, 230 (1950) 591-593

[7] É. Borel, Oeuvres d'É. Borel, Vol. 2, Éditions du CNRS, Paris, 1972, 1203-1204.

[8] Peter Borwein, "On the Irrationality of Certain Series," Mathematical Proceedings of the Cambridge Philosophical Society, vol. 112 (1992), pp. 141-146.

[9] D. G. Champernowne, "The Construction of Decimals Normal in the Scale of Ten," Journal of the London Mathematical Society, vol. 8 (1933), pg. 254-260.

[10] R. Crandall and C. Pomerance, Prime Numbers: A Computational Perspective, SpringerVerlag, New York, 2002.

[11] R. Crandall and S. Wagon, "Sums of squares: computational aspects," manuscript, 2002. 
[12] D. Duverney, Keiji. Nishioka, Kumiko Nishioka, and I. Shiokawa, "Transcendence of Jacobi's theta series and related results," pp. 157-168, in Number Theory. Diophantine, Computational and Algebraic Aspects, Kálmän Yöry (ed.) et al. , Walter de Gruyter, Berlin, 1998.

[13] P. Erdős, "On Arithmetical Properties of Lambert Series," Journal of the Indian Mathematical Society (N.S.), vol. 12 (1948), pp. 63-66.

[14] P. Flajolet and I. Vardi, "Zeta Function Expansions of Classical Constants," manuscript, 1996, available at http://pauillac.inria.fr/algo/flajolet/Publications/Landau.ps

[15] E. Grosswald, Representations of Integers as Sums of Squares, Springer-Verlag, New York, 1985.

[16] H. Halberstam and H.-E. Richert, Sieve Methods, Academic Press, London, 1974.

[17] G. H. Hardy and E. M. Wright, An Introduction to the Theory of Numbers, Oxford University Press, 1979

[18] G. H. Hardy, Ramanujan: Twelve Lectures on Subjects Suggested by His Life and Work, 3rd ed. New York: Chelsea, 1999, pp. 9-10, 55, and 60-64.

[19] Aubrey J. Kempner, "On Transcendental Numbers," Transactions of the American Mathematical Society, vol. 17 (1916), pp. 476-482.

[20] M. J. Knight, “An 'Ocean of Zeroes' Proof That a Certain Non-Liouville Number is Transcendental," American Mathematical Monthly, vol. 98 (1991), pp. 947-949.

[21] L. Kuipers and H. Niederreiter, Uniform Distribution of Sequences, Wiley-Interscience, New York, 1974

[22] J. Lagarias, "On the Normality of Fundamental Constants," Experimental Mathematics, vol. 10, no. 3 (2001), pp. 353-366.

[23] E. Landau, Handbuch der Lehre von der Verteilung der Primzahlen, Bd. II, 2nd ed. New York: Chelsea, pp. 641-669, 1953.

[24] S. Lehr, "Sums and rational multiples of $q$-automatic sequences are $q$-automatic," Theor. Comp. Sci. 108 (1993) 385-391.

[25] S. Lehr, J. Shallit and J. Tromp, "On the vector space of the automatic reals," Theoretical Computer Science, vol. 163 (1996), pp. 193-210.

[26] J. H. Loxton, "A method of Mahler in transcendence theory and some of its applications," Bulletin of the Australian Mathematical Society, vol. 29 (1984), pp. 127-136.

[27] J. H. Loxton and A. J. van der Poorten, "Arithmetic properties of certain functions in several variables III," Bull. Austral. Math. Soc. 16 (1977), 15-47

[28] G. Martin, "Absolutely Abnormal Numbers," American Mathematical Monthly, vol. 108 (2001), no. 8, pp. 746-754.

[29] W. Miller, "Transcendence measures by a method of Mahler," Journal of the Australian Mathematical Society (Series A), vol. 32 (1982), pp. 68-78.

[30] Yu. V. Nesterenko, "Modular functions and transcendence questions," Mat. Sb. 187 (1996) 65-96; translation in Sb. Math. 187 (1996) 1319-1348 [MR 97m:11102]

[31] I. Niven, Irrational Numbers, Carus Mathematical Monographs, no. 11, Wiley, New York, 1956.

[32] P. Ribenboim, The New Book of Prime Number Records, Springer-Verlag, New York, 1996

[33] K. Roth, "Rational Approximations to Algebraic Numbers," Mathematika, vol. 2 (1955), pp. 1-20. Corrigendum, pp. 168.

[34] J. Samborski, Problem E2667, American Mathematical Monthly, vol. 84 (1977), pp. 567.

[35] J. Shallit, private communication.

[36] J. Shallit and A. van der Poorten, Canadian Journal of Mathematics, vol. 45 (1993), pp. 1067-1079.

David H. BAILEY

Lawrence Berkeley National Laboratory

1 Cyclotron Road

Berkeley, CA 94720, USA

E-mail : dhbailey@lbl.gov 
Jonathan M. BoRweIN

Department of Mathematics

Simon Fraser University

Burnaby, BC V5A 156, Canada

E-mail : jborwein@cecm.sfu.ca

Richard E. CRANDALL

Center for Advanced Computation

Reed College

Portland, OR 97202

E-mail : crandall@reed.edu

Carl Pomerance

Bell Laboratories

600 Mountain Avenue

Murray Hill, NJ 07974

E-mail : carlp@research.bell-labs.com 